NASA/TM-2002-210938

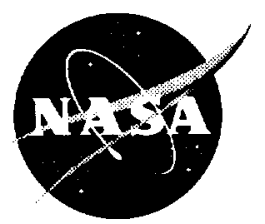

\title{
Mobile Router Technology Development
}

William D. Ivancic

Glenn Research Center, Cleveland, Ohio

David H. Stewart

Verizon, Federal Network Systems, LLC, Cleveland, Ohio

Terry L. Bell

Lockheed Martin Global Telecommunications, Cleveland, Ohio

Brian A. Kachmar

Analex Corporation, Brook Park, Ohio

Dan Shell and Kent Leung

Cisco Systems, San Jose, California 
Since its founding, NASA has been dedicated to the advancement of aeronautics and space science. The NASA Scientific and Technical Information (STI) Program Office plays a key part in helping NASA maintain this important role.

The NASA STI Program Office is operated by Langley Research Center, the Lead Center for NASA's scientific and technical information. The NASA STI Program Office provides access to the NASA STI Database, the largest collection of aeronautical and space science STI in the world. The Program Office is also NASA's institutional mechanism for disseminating the results of its research and development activities. These results are published by NASA in the NASA STI Report Series, which includes the following report types:

- TECHNICAL PUBLICATION. Reports of completed research or a major significant phase of research that present the results of NASA programs and include extensive data or theoretical analysis. Includes compilations of significant scientific and technical data and information deemed to be of continuing reference value. NASA's counterpart of peerreviewed formal professional papers but has less stringent limitations on manuscript length and extent of graphic presentations.

- TECHNICAL MEMORANDUM. Scientific and technical findings that are preliminary or of specialized interest, e.g., quick release reports, working papers, and bibliographies that contain minimal annotation. Does not contain extensive analysis.

- CONTRACTOR REPORT. Scientific and technical findings by NASA-sponsored contractors and grantees.
- CONFERENCE PUBLICATION. Collected papers from scientific and technical conferences, symposia, seminars, or other meetings sponsored or cosponsored by NASA.

- SPECIAL PUBLICATION. Scientific, technical, or historical information from NASA programs, projects, and missions, often concerned with subjects having substantial public interest.

- TECHNICAL TRANSLATION. Englishlanguage translations of foreign scientific and technical material pertinent to NASA's mission.

Specialized services that complement the STI Program Office's diverse offerings include creating custom thesauri, building customized databases, organizing and publishing research results ... even providing videos.

For more information about the NASA STI Program Office, see the following:

- Access the NASA STI Program Home Page at http://wwww.sti.nasa.gov

- E-mail your question via the Internet to help@sti.nasa.gov

- Fax your question to the NASA Access Help Desk at 301-621-0134

- Telephone the NASA Access Help Desk at 301-621-0390

- Write to: NASA Access Help Desk NASA Center for AeroSpace Information 7121 Standard Drive Hanover, MD 21076 
NASA/TM-2002-210938

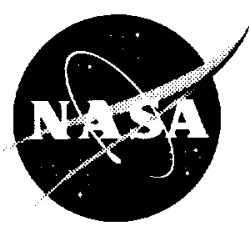

\section{Mobile Router Technology Development}

William D. Ivancic

Glenn Research Center, Cleveland, Ohio

David H. Stewart

Verizon, Federal Network Systems, LLC, Cleveland, Ohio

Terry L. Bell

Lockheed Martin Global Telecommunications, Cleveland, Ohio

Brian A. Kachmar

Analex Corporation, Brook Park, Ohio

Dan Shell and Kent Leung

Cisco Systems, San Jose, California

National Aeronautics and

Space Administration

Glenn Research Center 
Trade names or manufacturers' names are used in this report for identification only. This usage does not constitute an official endorsement, either expressed or implied, by the National Aeronautics and Space Administration.

Available from

NASA Center for Aerospace Information 7121 Standard Drive

Hanover, MD 21076
National Technical Information Service 5285 Port Royal Road Springfield, VA 22100

Available electronically at http://gltrs.grc.nasa.gov 


\title{
Mobile Router Technology Development
}

\author{
William D. Ivancic \\ National Aeronautics and Space Administration \\ Glenn Research Center \\ Cleveland, Ohio 44135 \\ rivanciciagrenasa gov, 216-433-3494 \\ David H. Stewart \\ Verizon, Federal Network Systems, LLC \\ Cleveland, Ohio 44135 \\ Divid.Stewaralerenast.gos, 216-433-9644 \\ Terry L. Bell \\ Lockheed Martin Global Telecommunications \\ Cleveland, Ohio 44135 \\ remp. Bellourenssigoy, 216-433-3725 \\ Brian A. Kachmar \\ Analex Corporation \\ Brook Park, Ohio 44142 \\ Brian. A. Kachmaragrc.nasa.go4, 216-433-8655 \\ Dan Shell and Kent Leung \\ Cisco Systems \\ San Jose, California 95134-1706 \\ dshellacisco.com, 2 16-781-0507 \\ hlem!racisco.com, 408-526-5030
}

Abstract-Cisco Systems and NASA have been performing joint research on mobile routing technology under a NASA Space Act Agreement. Cisco developed mobile router (MR) technology and provided that technology to NASA for applications to aeronautic and space-based missions. NASA has performed stringent performance testing of the MR, including the interaction of routing and transport-level protocols. This paper describes mobile routing, the MR, and some key configuration parameters. In addition, the paper describes the mobile routing test network and test results documenting the performance of transport protocols in dynamic routing environments.

\section{INTRODUCTION}

Cisco Systems and NASA Glenn Research Center have been performing joint networking research under a NASA Space Act Agreement to apply Internet technologies and protocols to aeronautic and space-based communications. Cisco Systems developed the mobile router (MR) during this time and provided the technology for both the commercial and government markets. The technology was given to NASA for evaluation of its applicability to NASA's aeronautic and space-based networks. By performing joint testing of the MR, NASA was able to provide input about applications and requirements. Cisco was then able to modify the code where appropriate to address those needs and requirements. This paper will describe mobile routing, the $\mathrm{MR}$ and some key configuration parameters, the test network, and the performance of transport protocols in dynamic routing environments.

\section{MOBILE IP OVERVIEW}

Mobile IP (ref. 1) is a routing protocol that allows hosts (and networks) to seamlessly "roam" among various IP subnetworks. This is essential in many wireless networks. Mobile IP can also be useful in wireless networks where the MN's point of attachment to the network changes due to varying conditions in the wireless medium, even if the MN is not physically moving. In a wired network, mobile IP can be used where the MN simply wishes to maintain its network identity, since the MN is always contacted through association of its home IP address. 
There are three basic elements in the mobile IP: the home agent (HA), the foreign agent (FA), and the mobile node (MN).

"The Home-agent $(\mathrm{HA})$ is a router on a mobile node's home network that tunnels datagrams for delivery to the mobile node when it is away from home, and maintains current location information for the mobile node. The home-agent is the mobile node's gateway to the network. All traffic destined for the mobile node must pass through the home-agent.

"The Foreign-agent (FA) is a router on a remote network that provides routing services to a registered mobile node. The foreign-agent receives datagrams from the home-agent via an IP tunnel, which are then forwarded to the mobile node. For datagrams sent by a registered mobile node, the foreign-agent serves as a default router.

"The Mobile-node (MN) is a host or router that changes its point of attachment from one network or subnetwork to another. A mobile node may change its location without changing its IP address; it may continue to communicate with other Internet nodes at any location using its (constant) IP address, assuming link-layer connectivity to a point of attachment is available." (ref. 1)

An MN first determines whether it is attached to its home network or a foreign network using Internet control message protocol (ICMP) router discover messages. If it is attached to a foreign network, the MN will determine if an FA is available. If so, the MN registers with the HA via the FA, and a unidirectional IP tunnel ${ }^{1}$ is established from the HA to the FA. The HA will now encapsulate all packets destined for the MN and tunnel them to the FA. The FA de-encapsulates the packets and forwards them on to the MN. Standard IP routing is used to deliver datagrams sent by the MN, with the FA as the MN's default router.

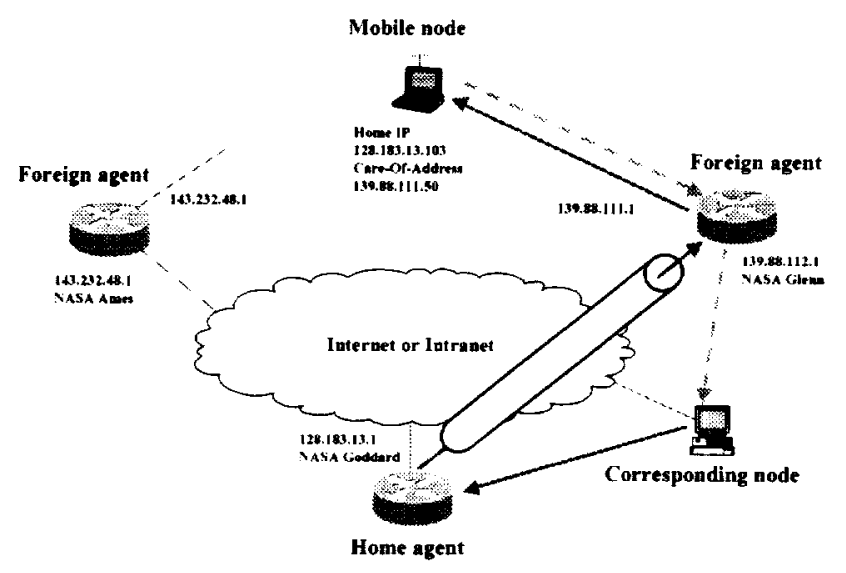

Figure 1. Mobile IP Tunneling

Figure 1 depicts mobile-IP operation when the MN is a single host. In this scenario, the corresponding node requests information from the MN. The request is sent first to the MN's home IP address via the Internet. Thus, the request goes to the HA. The HA encapsulates the request in a second IP packet and tunnels that request through the Internet to the FA. The FA de-encapsulates the request and passes it on to the MN via the care-of address ${ }^{2}$ (COA). Once the $M N$ receives the request, it sends a reply to the corresponding node through normal IP routing. Thus, the reply goes to the FA, which passes it on to the corresponding node via the Internet.

\footnotetext{
I Tunneling is a technique that enables one nerwork to send its data via another network's connections. Tunneling works by encapsulating a network protocol within packets carried by the second network.

2 It is also possible to have a collocated COA. In the case of a collocated COA, the HA encapsulates packets directly to the MN instead of to the FA.
} 


\section{MOBILE ROUTER OVERVIEW}

The MR is software code that resides in a network router. It is part of the mobile IP standards specification. An MR allows entire network(s) to roam. Hence, a device connected to the MR does not need to be an MN because the MR is providing the roaming capabilities.

Cisco Systems has recently implemented MR code. NASA Glenn and Cisco, through a Space Act Agreement, have been performing joint research and validation tests on this code, which became part of the Cisco Systems Internetworking Operating System (IOS) in the second half of 2000. The MR is currently being deployed for a static network, which only supports stub routers. Future implementations will support dynamic networks.

MR code allows a router to be an MN. The difference between a mobile host and an MR is that once the MR has registered with the HA, the HA will inject the MR's networks into the HA's routing table and redistribute these routes. This implementation is fully compliant to the RFC 2002 standard. No alteration to the specification is necessary to achieve this static networking.

Figure 2 depicts mobile-IP operation when the MN is a network. The operation is nearly identical to that of a mobile IP for a single host. The exceptions are that two tunnels are established: one between the HA and FA (Tunnel1), and one between the HA and MR (Tunnel0). The HA will perform two encapsulations of any packet destined for the MR and forward all packets for the mobile network to the FA. The FA will perform one de-encapsulation and pass the packets to the MR. The MR performs the second de-encapsulation and forwards the packets to the devices on its networks. As the MR moves, it will register its whereabouts with its HA via various FA's. Thus, an MR is an MN; however, the node is a network rather than a single host.

The MR has a number of features that make it applicable to a variety of networks and applications.

- The MR can have multiple roaming interfaces. This allows for connection to a variety of different wired and wireless links.

- The MR can perform smooth handoffs, preferred path routing, and priority routing.

- The HA can be configured for hot-standby. This is extremely important. If the HA were to experience a catastrophic failure, all routes would be lost. Hot-standby was demonstrated by pulling the power plug on the active HA and noting restoration time. Restoration was nearly instantaneous.

- The mobile routing protocols perform well in long delay environments. Testing of the MR capabilities over links with round-trip time delays in excess of $3 \mathrm{sec}$ was performed. This delay is far greater than a double geostationary satellite hop.

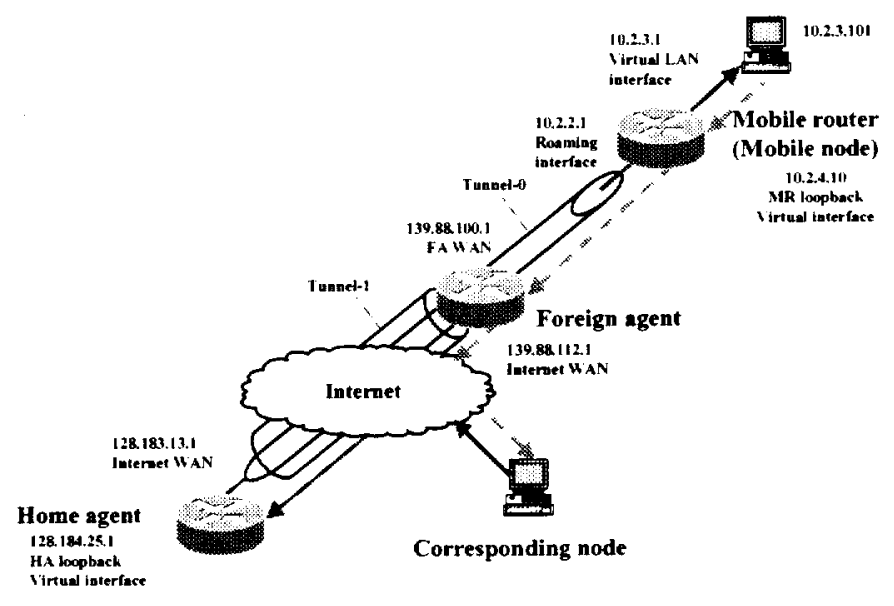

Figure 2. Mobile Router Tunneling 
As of April 2001, Internet protocol security (IPSec) ${ }^{3}$ between the FA and MR, multicasting, and unidirectional link routing (UDLR) ${ }^{+}$were still being addressed. Currently, security between the MR and HA is performed using the 128-bit message digest algorithm (MD5). Security can also be implemented between the FA and HA via IPSec and/or by the establishment of access lists in the HA.

\section{Mobile Routing Operations}

\subsection{AGENT DISCOVERY}

Agent discovery is a key function of mobile routing, both for mobile hosts and MR's. For many network architectures that utilize MR's, optimal configuration of agent discovery and advertisement could result in improved system performance or lower communication costs. Therefore, agent discovery will be discussed in detail here. Issues include

- Speed of FA discovery (initial registration)

- Who pays for the Wide-Area Network (WAN) RF bandwidth

- Cost of RF bandwidth

System performance and communication cost optimization is performed by adjustment of various timers in the MR and FA, as well as by enabling or disabling FA advertisements and MR solicitations. Having the MR periodically solicitate or decreasing the interval between the FA advertisments will speed up FA discovery at the expense of increased bandwidth utilization. This may not be desirable for expensive or bandwidth-limited wireless connections. Thus, great care should be utilized when configuring the mobile network.

An MR discovers an FA by receiving agent advertisements on its interfaces configured for roaming. Agent solicitation may also be configured on an MR interface that does not expect periodic agent advertisements from FA's.

An MR will send out agent solicitations to find FA's

- If the interface is configured for roaming

- If the MR's roaming interface comes up

- If there is a retransmission after no advertisement is heard

- Before advertisement expiration

A table of available FA's and their associated COA's are derived from agent advertisements heard on the MR's roaming interfaces. The MR then registers with the HA through one of the FA's listed in this table. The FA that the MR selects is known as the active FA, and the MR's connection to the active FA is known as the preferred path.

The selection of the active FA and preferred path is based on the MR's roaming interfaces. If more than one interface receives agent advertisements, the one with the higher roaming priority value is selected as active. If multiple interfaces have the same priority, the highest bandwidth is active. If interfaces have the same bandwidth, the highest IP address is active. The MR will then send a registration request bound for the HA to the active FA via the preferred path.

A learned FA entry is removed from the table when

- An unsolicited interface's advertisement ages out

- Solicitation retransmissions max out

- Roaming on the interface is deconfigured

- Registration via an active FA to the HA ages out

\footnotetext{
${ }^{3}$ The IPSec protocol provides secure, interoperable communication across a network, transparent to the application.

${ }^{4}$ UDLR is a mechanism to emulate bidirectional connectivity between nodes that are directly connected by a unidirectional link.
} 
- Unrecoverable registration denials are received from HA

- The MR is disabled

- The interface where the FA was learned goes down

- The user manually clears FA via the command line interface

There are a variety of timers available to optimize network performance. There are three timers in the MR: agent solicitation, agent advertisement, and registration. The agent solicitation timer is for periodic transmission of solicitations generated by the MR. The agent advertisement timer is for aging out received advertisements from an FA. Both the agent solicitation and agent advertisement timers are used as watchdog timers for the MR's agent discovery of an FA. The registration timer is for periodic transmission of registration requests to the HA and is used as a watchdog timer for the registration between the HA and the MR. There are timers within the FA to control the FA advertisments. These timers are associated with the ICMP router discovery protocol (IRDP) configuration and include the minimum and maximum advertisement intervals and the holdtime, the time for which advertisements are valid. One timer is available on the HA to globally control the maximum valid registration lifetimes of mobile hosts. This timer may also be configured in the FA and MR, but the lifetime of the registration will be set to the lesser of the 3 time settings.

\subsection{REGISTRATION}

After determining the preferred path, the MR will send a registration request to the HA. Since the MR is attached to a foreign network, the registration request is sent first to the active FA, which then forwards the request to the HA. Registration requests are only sent out to an active FA. The following events will trigger a registration request:

- If an FA advertisement is received from the MR and

- Movement from one FA to another has been detected

- An FA reboot has been detected

- The MR is currently isolated

- An advertisment has been received from another FA that is connecting via a better interface (preferred path)

- If an active FA advertisement (IRDP lifetime) expires, and the MR chooses another learned FA to reregister through

- If a registration timer expires due to retransmission or lifetime aging

- If the hold-down period on the FA expires

- If the interface connected to the active FA goes down, and there are other FA's

- If the MR configuration changes

- If the MR has to recover from HA denial due to mismatched ID

- If the MR has to recover from FA denial due to lower lifetime

\subsection{Key CONFIGURATIONS PARAMETERS}

Figure 3 shows the detailed network configuration used to test the MR at NASA Glenn. We will utilize this to describe the test network, as well as to help illustrate the configurations of the various mobile network routers: the $\mathrm{HA}$, the FA, and the MR.

\subsubsection{HOME AgENT CONFIGURATION}

The following is a partial listing of the HA configuration highlighting key configuration parameters.

A loopback interface is created for the HA network address. This is done so that any physical interface can go down without the HA being lost. The static routes and mobile subnetworks must be redistributed. This is done using the redistribute command in the router setup. The command "router mobile" enables the mobile-IP process on the router. 


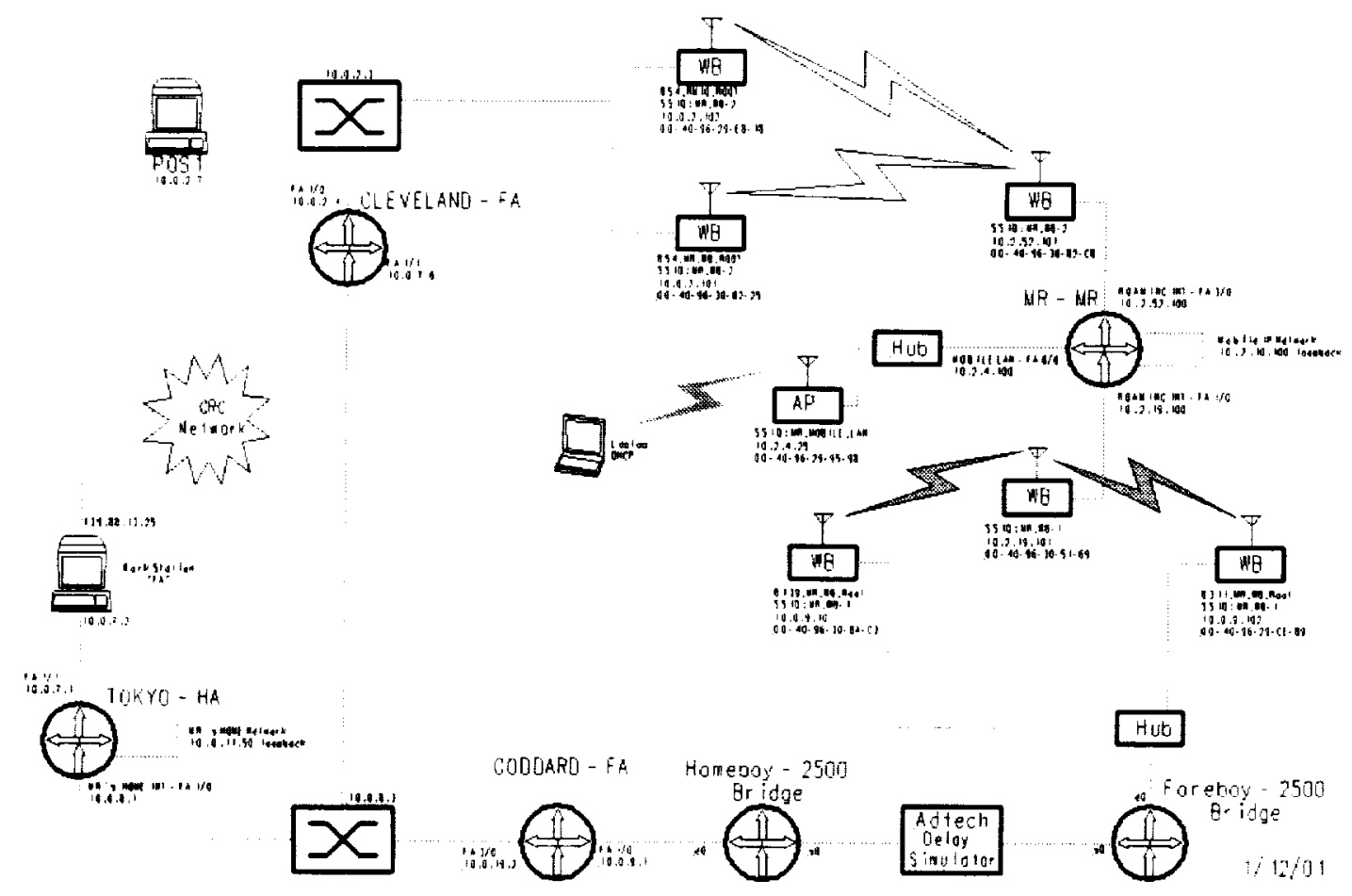

Figure 3. Mobile Router Test Network Configuration

A static route has been established to enable routing from our unroutable network to the Glenn network via a linux workstation configured to forward packets. This enables us to route to the Internet for demonstrations but to disconnect for all other testing, thereby maintaining a secure network.

The MR-specific configuration commands using the "IP mobile" commands. A virtual network is the first setup. Here the virtual network is 10.2.0.0. Next, the mobile networks are established. These are the loopback and physical interfaces on the MR. The mobile host is then defined as is the MD5 security association.

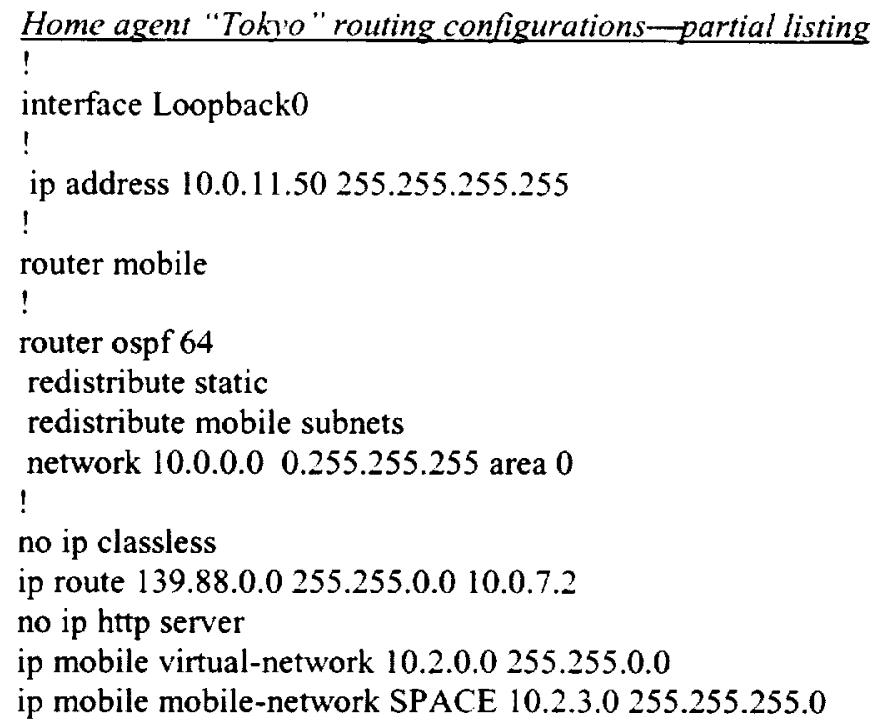


ip mobile mobile-network SPACE 10.2.52.0 255.255.255.0

ip mobile mobile-network SPACE 10.2.4.0 255.255.255.0

ip mobile mobile-network SPACE 10.2.10.0 255.255.255.0

ip mobile mobile-network SPACE 10.2.19.0 255.255.255.0

ip mobile host 10.2.10.100 mobile-network SPACE virtual-network 10.2.0.0 255.255.0.0

ip mobile secure host 10.2 .10 .100 spi 500 key hex 12345678123456781234567812345678

!

The routing tables, bindings, and tunnels for the HA are shown below. Note that all the networks on the MR are connected via Tunnell, whereas the address assigned to the MR (MN) is connected via Tunnel0. The bindings show that the MR is bound to the HA through FA 10.0.8.22 and that the COA is 10.0.2.1. The bindings also show a registration lifetime of $35 \mathrm{sec}$. In this case, the lifetime count was taken from the MR because the MR requested lifetime is less than the HA default of $10 \mathrm{hr}$.

Home agent "Tohvo" diagnostic displavs—select displays

TOKYO\#sho ip route

Codes: C - connected, S - static, I - IGRP, R - RIP, M - mobile, B - BGP

D - EIGRP, EX - EIGRP external, O - OSPF, IA - OSPF inter area

N1 - OSPF NSSA external type 1, N2 - OSPF NSSA external type 2

E1 - OSPF external type 1, E2 - OSPF external type 2, E - EGP

i - IS-IS, L1 - IS-IS level-1, L2 - IS-IS level-2, ia - IS-IS inter area

* - candidate default, U - per-user static route, o - ODR

$P$ - periodic downloaded static route

Gateway of last resort is not set

S $\quad 139.88 .0 .0 / 16[1 / 0]$ via 10.0 .7 .2

$10.0 .0 .0 / 8$ is variably subnetted, 14 subnets, 3 masks

M $\quad 10.2 .10 .0 / 24[3 / 1]$ via 10.2.10.100,3w5d, Tunnel 1

C $\quad 10.0 .8 .0 / 24$ is directly connected, FastEthernet $1 / 0$

O $\quad 10.0 .9 .0 / 24[110 / 85]$ via $10.0 .8 .25,3 w 5 \mathrm{~d}$, FastEthernet $1 / 0$

O $\quad 10.0 .2 .0 / 24[110 / 2]$ via 10.0.8.22, 3 w5 d, FastEthernet $1 / 0$

$\mathrm{M} \quad 10.2 .0 .0 / 16$ is directly connected

M 10.2.3.0/24[3/1] via 10.2.10.100,3w5d, Tunnel 1

M $\quad 10.2 .4 .0 / 24[3 / 1]$ via $10.2 .10 .100,3 w 5 \mathrm{~d}$, Tunnell

C 10.0.7.0/24 is directly connected, FastEthernet $1 / 1$

O $\quad 10.0 .19 .0 / 24[110 / 75]$ via $10.0 .8 .25,3 w 5 d$, FastEthernet $1 / 0$

M 10.2.19.0/24[3/1] via 10.2.10.100,3w5d, Tunnell

O $\quad 10.0 .20 .0 / 24[110 / 65]$ via 10.0.8.25.3w5d, FastEthernet $1 / 0$

C $\quad 10.0 .11 .50 / 32$ is directly connected, Loopback 0

M 10.2.52.0/24 [3/1] via 10.2.10.100,3w5d, Tunnell

M $\quad 10.2 .10 .100 / 32[3 / 1]$ via $10.0 .2 .1,3 w 5 \mathrm{~d}$, Tunnel0

TOKYO\#sho ip mobile tunnel

Mobile Tunnels:

Tunnel0:

src 10.0.11.50, dest 10.0.2.1

encap IP/IP, mode reverse-allowed, tunnel-users 1

IP MTU 1480 bytes

Path MTU Discovery, mtu: 0, ager: 10 mins, expires: never

outbound interface FastEthernet $1 / 0$

HA created, fast switching enabled, ICMP unreachable enabled

0 packets input, 0 bytes, 0 drops

0 packets output, 0 bytes 
Tunnell:

src 10.0.11.50, dest 10.2.10.100

encap IP/IP, mode reverse-allowed, tunnel-users 1

IP MTU 1460 bytes

Path MTU Discovery, mtu: 0, ager: 10 mins, expires: never

outbound interface Tunnel0

HA created, fast switching enabled, ICMP unreachable enabled

0 packets input, 0 bytes, 0 drops

0 packets output, 0 bytes

Mobility Binding List:

Total 1

10.2.10.100:

Care-of Addr 10.0.2.1, Src Addr 10.0.8.22

Lifetime granted 00:00:35 (35), remaining 00:00:20

Flags sbdmgvt, Identification BDFEA112.4D6IDAFC

Tunnel0 src 10.0.11.50 dest 10.0.2.1 reverse-allowed

MR Tunnelo sre 10.0.11.50 dest 10.0.2.1 reverse-allowed mobile-network SPACE

Routing Options -

\subsubsection{FOREIGN AGENT CONFIGURATION}

The following is a partial listing of the configuration of one of the FA's (CLEVELAND), highlighting key configuration parameters.

ICMP router discovery protocol (IRDP) must be running on the FA interfaces. Here, that interface is 10.0 .2 .1 . For this configuration, the FA will advertise its services every 7 to $10 \mathrm{sec}$ as defined by the minimum and maximum advertisement intervals. The IRDP holdtime indicates to the MR the period of time (in sec) that the FA's advertisement is valid. In the event that the MR does not receive an advertisement from the active FA before the holdtime timer expires, MR assumes that it is no longer on the active FA's network, and will attempt to re-register with an FA in its table.

The mobile registration lifetime for the FA 10.0 .2 .1 is set for $40 \mathrm{sec}$. Setting the registration lifetime on the FA limits the maximum lifetime of registration that the FA will allow the MR to request from the HA. This parameter may also be configured in the HA and MR. However, the lifetime of the registration will be set to the shortest of the three time settings.

As with the HA, the "router mobile" command must be issued to initiate the mobile-IP process.

FA capability is initialized on each interface via the "IP mobile foreign-service" command. In our case, only interface 10.0.2.1 is acting as an FA.

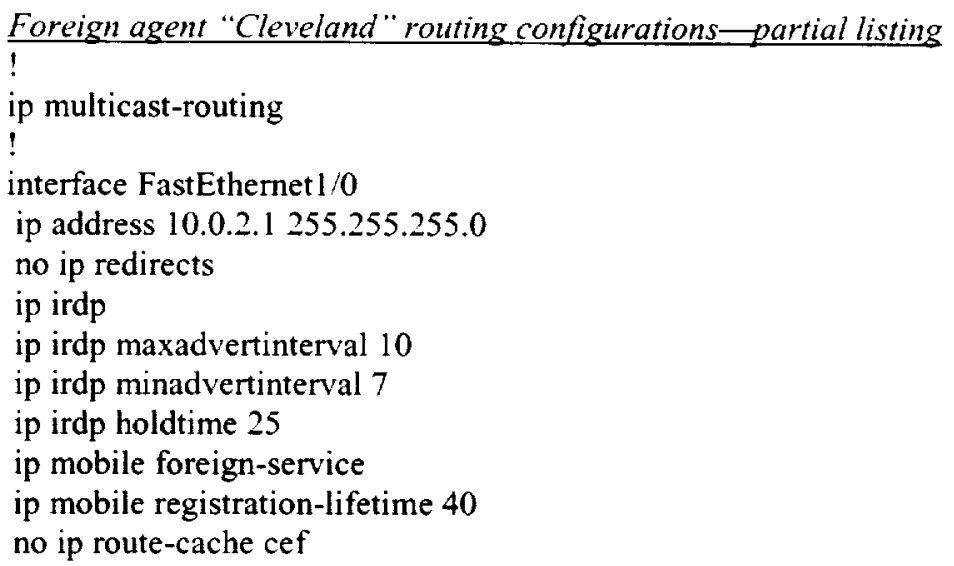




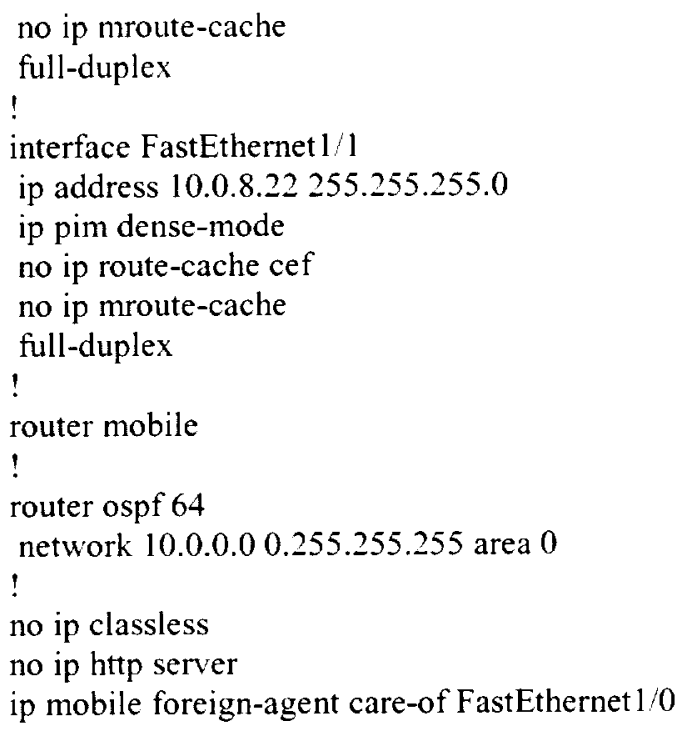

A listing of the FA visitors is shown below. There is only one visitor, the MR, as identified by the source address, 10.2.10.100. The MR's registration lifetime with the HA is $35 \mathrm{sec}$.

\section{Foreign agent "Cleveland" diagnostic displavs-select displays}

CLEVELAND\#sho ip mobile visitor

Mobile Visitor List:

Total 1

10.2.10.100:

Interface FastEthernet 1/0, MAC addr 0002.4b47.7d70

IP src 10.2.10.100, dest 10.0.2.1, UDP src port 434

HA addr 10.0.11.50, Identification BE0006B8.B330DDD8

Lifetime 00:00:35 (35) Remaining 00:00:22

Tunnel0 src 10.0.2.1, dest 10.0.11.50, reverse-allowed

Routing Options -

\subsubsection{MOBILE ROUTER CONFIGURATION}

The following is a partial listing of the MR configuration, highlighting key configuration parameters:

As with the HA, a loopback interface is created for the MR network address. This is done so that any physical interface can go down without the entire MR being lost.

The MR has four active physical interfaces. For the test network shown in figure 3, only the three Ethernet interfaces are being used. One of the Ethernet interfaces, FastEthernet0/0, is the LAN, while the other interfaces are configured as mobile interfaces that would connect to FA's. This is defined with the "IP MR-service roam" command. Priorities can also be set for each roaming interface, thereby establishing preferred service paths.

Routing protocols should be running on the MR if the MR ever plans to return home. When the MR is back home, it needs to propagate its mobile network to its HA using a routing protocol such as the open shortest path first (OSPF) or the routing information protocol (RIP). For this particular configuration, we are using OSPF.

The "router mobile" command is issued to enable the mobile-IP process.

The HA address has to be defined and proper security associations must be established between the HA and MR. This is accomplished with the "IP mobile secure home agent" command. 
The "IP mobile router" command is used to enable the mobile routing functions, to set the address of the MR, and to set registration lifetimes and retransmission timers.

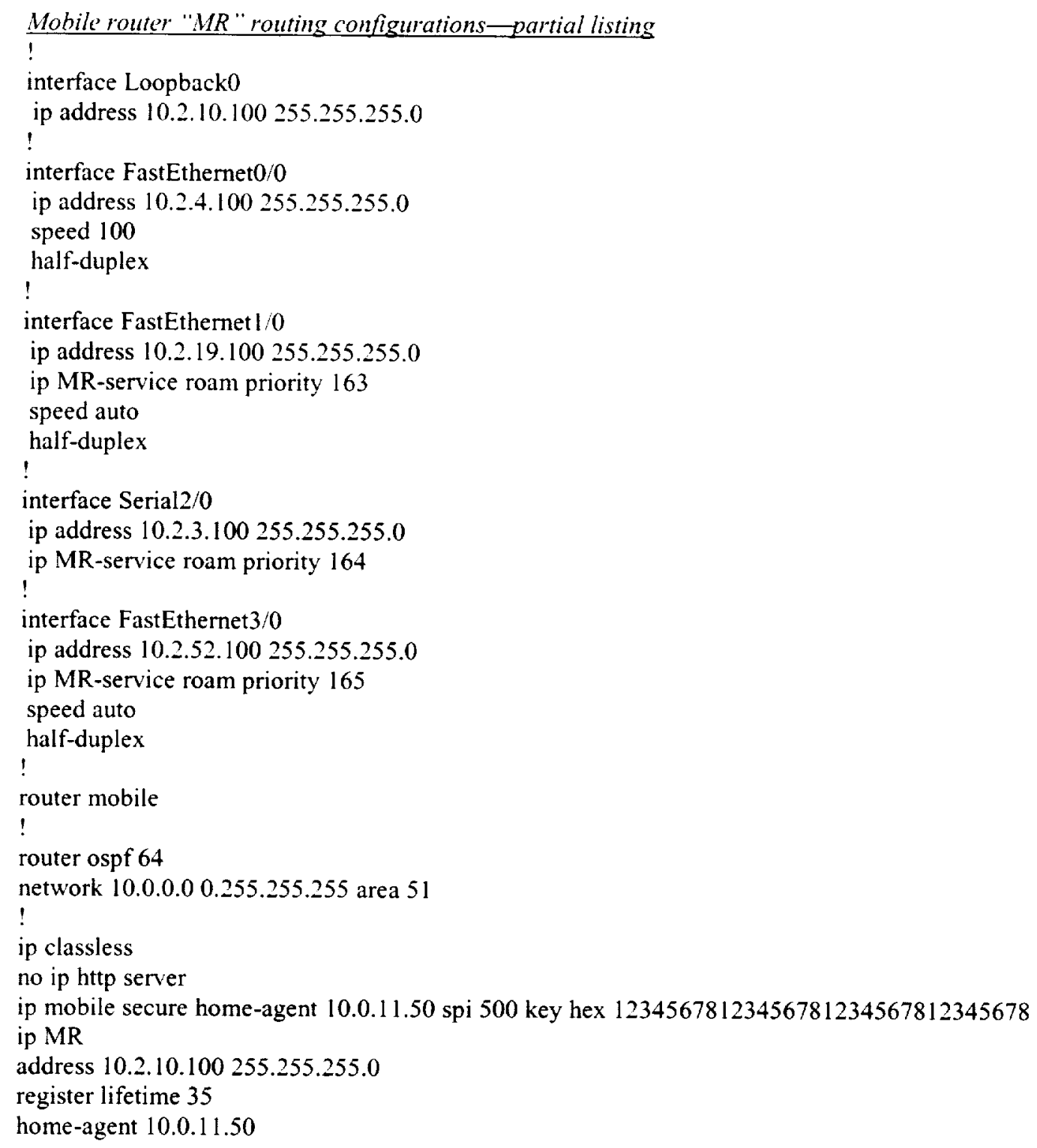

The routing table for the MR shows the physical interfaces as all directly connected. The FA 10.0.2.1 is the mobile connection and is set as the default router. Basically, the default router will always be the connection of the best available (preferred path) FA.

There is currently only one FA registered with the MR. From the "show IP MR agent" command, we can see that the FA is 10.0.2.1 with a registration lifetime of $40 \mathrm{sec}$.

Mobile router "MR" diagnostic displavs-select displays

Mobile-Router\#sho ip route

Codes: C - connected, S - static, I - IGRP, R - RIP, M - mobile, B - BGP

D - EIGRP, EX - EIGRP external, O - OSPF, IA - OSPF inter area

N1 - OSPF NSSA external type 1, N2 - OSPF NSSA external type 2

El - OSPF external type 1, E2 - OSPF external type 2, E - EGP 
i - IS-IS, L1 - IS-IS level-1, L2 - IS-IS level-2, ia - IS-IS inter area

* - candidate default, U - per-user static route, o - ODR

$P$ - periodic downloaded static route

Gateway of last resort is 10.0.2.1 to network 0.0.0.0

10.0.0.0/8 is variably subnetted, 5 subnets, 2 masks

C 10.2.10.0/24 is directly connected, Loopback 0

M $\quad 10.0 .2 .1 / 32[3 / 1]$ via 10.0.2.1,3w5d, FastEthernet3/0

C 10.2.4.0/24 is directly connected, FastEthernet $0 / 0$

C 10.2.19.0/24 is directly connected, FastEthernet $1 / 0$

C $\quad 10.2 .52 .0 / 24$ is directly connected, FastEthernet $3 / 0$

$\mathrm{M}^{*} \quad 0.0 .0 .0 / 0[3 / 1]$ via $10.0 .2 .1,3 \mathrm{w} 5 \mathrm{~d}$, FastEthernet $3 / 0$

Mobile-Router\#sho ip MR agent

MR Agents:

FA 10.0.2.1:

Care-of address 10.0.2.1

Interface FastEthernet $3 / 0$, MAC $0010.0 \mathrm{~b} 4 \mathrm{a} .7020$

Agent advertisement seq 26450, Flags rbhFmGvt, Lifetime 40

IRDP advertisement lifetime 25 , Remaining 22

Last received 03/28/93 18:45:13

First heard 03/02/93 03:32:55

\section{TEST NETWORK}

In order to test the MR in a relevant environment, a wired and wireless mobile testbed was developed at NASA Glenn. Figure 3 depicts the network configuration of that testbed. We have four mobile-IP-enabled routers: one HA, two FA's, and one MR. Two additional routers, "homeboy" and "foreboy," reside between the FA wireless access point. These two routers are configured as bridges and are there simply to provide a serial interface for a satellite channel emulator. A linux workstation is connected to the HA and performs network address translator (NAT) functions in order to allow routing from the 10.x.x.x network to the NASA Glenn network.

The satellite emulator is an Adtech SX/14 and provides both delay and error capability. We have used this unit in the MR testing to generate delay on the duplex path.

The MR resides in a half-rack roll-around cabinet (fig. 4), which can be placed in the laboratory or on a van. The router is a Cisco 3640 with one four-port serial network interface card (NIC) and three Ethernet NIC"s. Connections can be wired or wireless while in the laboratory, and wireless via 802.11 wireless Ethernet connections when run in the van. The MR LAN also has wireless capabilities as well as a wired hub. Dynamic host control protocol (DHCP) is running on the MR LAN in order to easily add application and test computers to the network. An uninterruptible power supply (UPS) allows for up to $2 \mathrm{hr}$ of mobile operation when no other power is available. In addition, the UPS cleans up the power provided by a diesel engine in the van.

There are four wireless 802.11 WAN connections, two for each FA. One of the wireless connection antennas is located in the laboratory and is used when the MR is being tested there. Three wireless connections are placed on various buildings throughout the Glenn Research Center. One wireless antenna connects to the Cleveland FA. The other two antennas are connected to the Goddard FA and have the programmable delay unit in the path between the FA and the MR. All connections are fiber-repeated back to the laboratory in order to keep the IP traffic off the production network. However, the production network will be moved as soon as performance testing is complete. 
Figure 5 shows a map of the antenna locations and a rough coverage map. The sites were picked such that we would have simultaneous coverage at some times, thereby enabling testing of smooth handoffs and preferred path operation.

Figure 6 shows the various antennas deployed throughout the Glenn Research Center and on the MR test van. Dipole antennas are used on the buildings to provide circular coverage. Both yagi and dipoles are used on the van. Only one set is deployed at any one time. The yagis provide greater gain, but are directional. The yagi antennas enable us to test at farther distances if we wish, or to restrict coverage and force handoffs and loss of signal by positioning the yagi antenna away from the reciprocal signal.

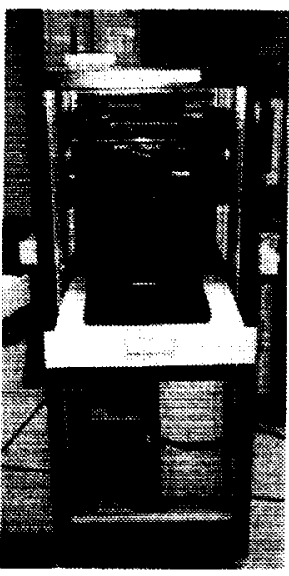

Figure 4. Mobile Router Rack

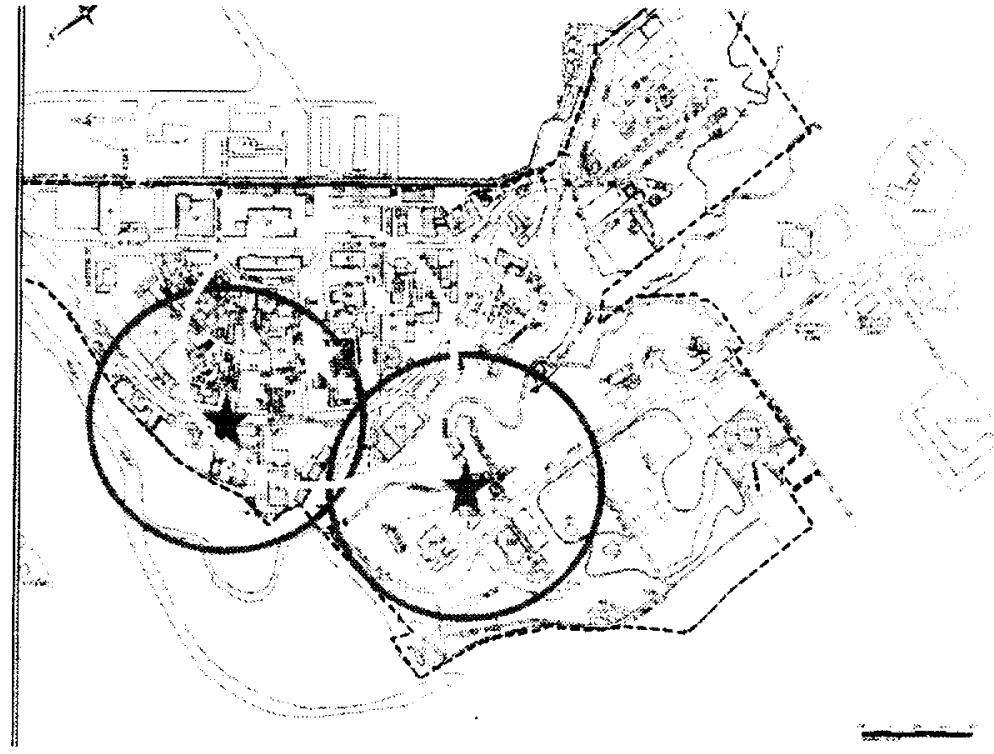

Figure 5. Wireless Ethernet Antenna Coverage
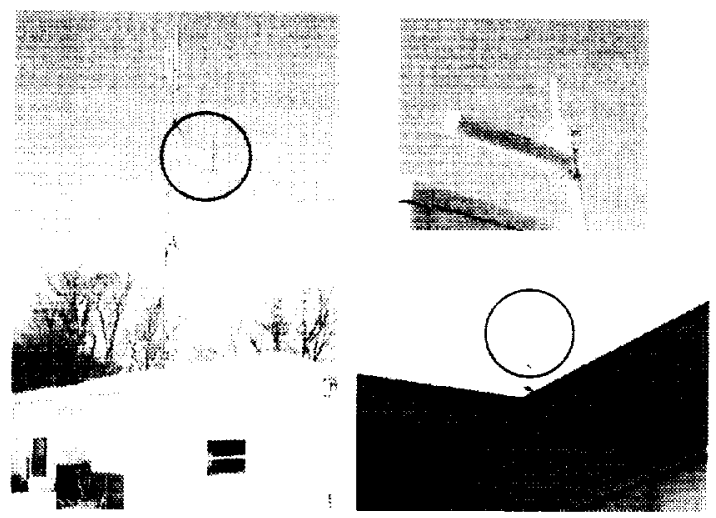

Figure 6. Dipole and Yagi Deployment 


\section{HANDOFFS AND HOLD-DOWN TIMERS}

The MR only allows one active route between itself and the HA. Whenever two or more paths are available for routing data between an MR and an HA, only one is selected as the preferred path. If two or more paths are available, the preferred path always takes precedence. In a wireless network, there are situations where the nonpreferred path has significant RF signal strength, yet the preferred path has sufficient RF signal strength to register but be continuously fading in and out. This results in a "flapping interface," whereby the MR is continuously setting up and tearing down routes to the preferred path as the RF signal fades in and out. The result is very poor data transfer, even though a strong signal and path is available from the nonpreferred path. In order to reduce this problem, a hold-down time is incorporated in the MR code and is set globally within the MR. The hold-down timer is used to establish a predefined amount of time that an interface must be stable before it is declared usable. In order to test the hold-down timer, we placed the van in a high-fade location. The preferred path had very little delay $(10$ to $40 \mathrm{msec}$ ) and $10 \mathrm{Mbps}$ bandwidth. The nonpreferred path had $700 \mathrm{msec}$ of delay and $128 \mathrm{kbps}$ of bandwidth.

Figures 7 and 8 show a TCP transmission for the same amount of data with the hold-down timer set to zero and $30 \mathrm{sec}$, respectively. In figure 7, the data is being transferred over the preferred path at all times, except during the initial $20 \mathrm{sec}$ of transmission. The slope of the time/sequence plot shows this. The nontransfer time where the TCP retransmission timers begin is due to the flapping interface. Total transmission time when the hold-down timer was set to zero was approximately $5 \mathrm{~min}$, even though the transmissions occurred almost exclusively over the $10 \mathrm{Mbps}$ link. In figure 8 , the hold-down timer was set to $30 \mathrm{sec}$. Nearly all the transmissions occurred over the 128 -kbps, 700-msec delay. Even with this lower bandwidth and additional delay, the same transmission only took a little over $2 \mathrm{~min}$. Approximately $30 \mathrm{sec}$ into transmission, the preferred path became active and then lost the signal. Thus, the $\mathrm{RF}$ signal strength for the preferred path was sufficiently stable for at least $30 \mathrm{sec}$ before fading. Flapping of the interface occurred, but the problem was significantly reduced. Increasing the hold-down time will improve the stability of the network. However, this also results in interfaces not being declared usable until the hold-down time expires. Thus, great care should be taken when setting this timer.

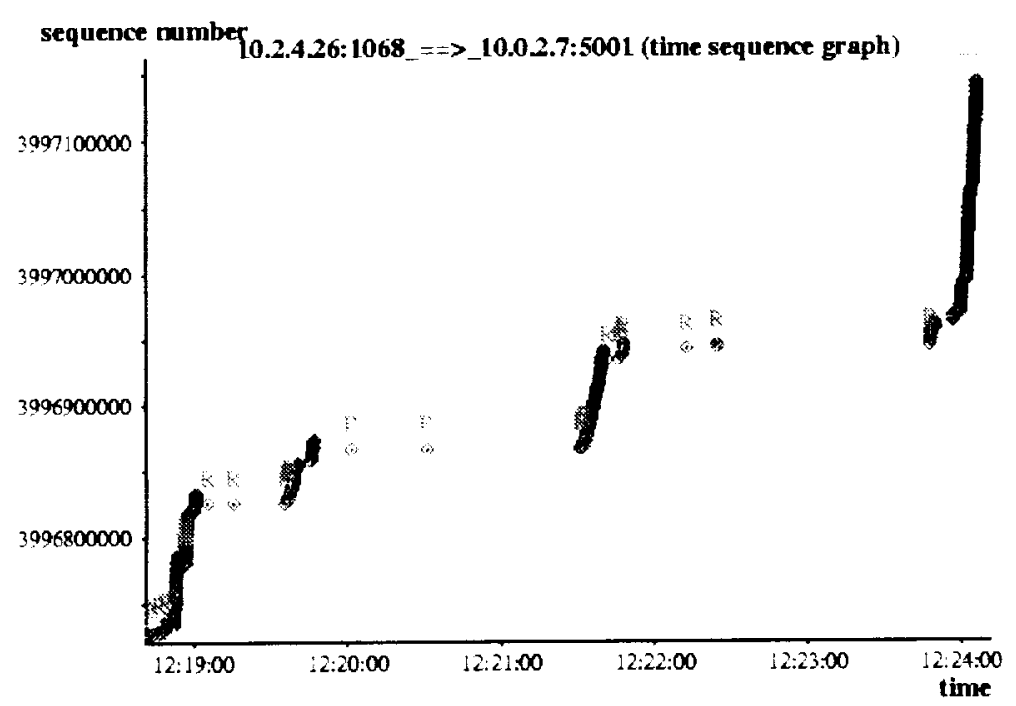

Figure 7. TCP Transfer with Hold-Down Timer $=0$ 


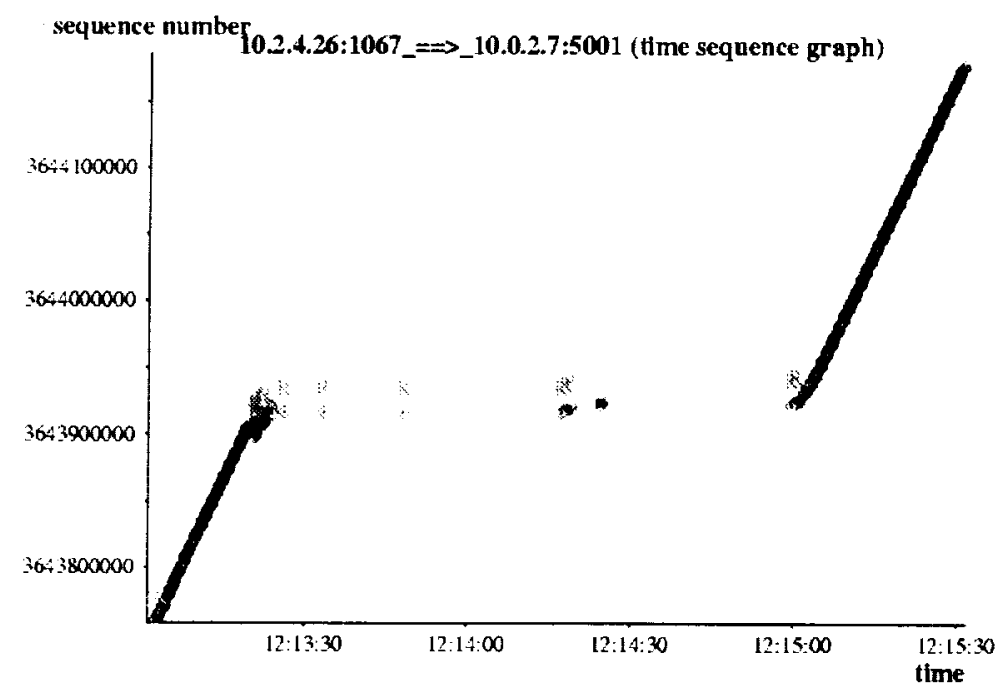

Figure 8. TCP Transfer with Hold-Down Timer $=30 \mathrm{sec}$

\section{TRANSPORT Protocol PERFormance}

We have demonstrated the MR functionality with live demonstrations. These live demonstrations validated the general mobile routing algorithms, including the preferred path. The MR was able to perform with round-trip time delays of up to $3 \mathrm{sec}(1.5 \mathrm{sec}$ in each link). Some of the applications we demonstrated included email transfers, Web browsing, voice-over IP (VOIP), ftp file transfers, secure shell, and Telnet.

In order to access the system performance during handoff situations, a series of tests was developed to emulate two operational networks: a terrestrial wireless network and an aeronautical wireless network. The testbed shown in figure 3 was used. The bandwidth on the preferred path was $10 \mathrm{Mbps}$ and the bandwidth on the nonpreferred path was $128 \mathrm{kbps}$. The only difference between the wireless terrestrial network and the aeronautical network was the delay settings of the Adtech SX/14. For the wireless network, we used $100 \mathrm{msec}$ round-trip times, and for the aeronautical network, we used $700 \mathrm{msec}$ round-trip times. We use $100 \mathrm{msec}$ as a reasonable estimate of terrestrial network delays through a wireless Internet service provider (ISP). The $700-\mathrm{msec}$ round-trip time includes $500 \mathrm{msec}$ for single-hop geostationary satellite round-trip delays and $200 \mathrm{msec}$ for terrestrial round-trip delays.

In order to isolate the handoff performance from other networking issues (congestion control, Quality-of-Service, etc.), data was taken for a single user emulation. The TCP implementations used were configured with fast retransmission, selective acknowledgement, and time-stamps options. The TCP performance tool ttcp was used. TCP receiver and transmitter buffer sizes were set for 11200 bytes, which is optimal for the bandwidth-delay product of the nonpreferred path at $700 \mathrm{msec}$ delay. Packet size was 1400 bytes. The hold-down timer was set for $30 \mathrm{sec}$. Tests were performed for both 100 and $700 \mathrm{msec}$ delays while the MR transitioned between nonpreferred and preferred paths. Two ttcp sessions were running simultaneously. The first had the source on the MR LAN and the destination on a LAN connected to an FA. The second had the source at the FA LAN and the destination on the MR LAN.

Figures 9 and 10 show simultaneous TCP transmissions between a workstation on an FA LAN and a workstation on an MR LAN. The delay in the nonpreferred, 128-kbps path was set to $100 \mathrm{msec}$. Data was taken at the source of the TCP transmission. The workstation clocks were not synchronized; therefore, there is a slight offset in the timing 
settings on the time-sequence plots. However, it is readily apparent that transmissions originating on the MR LAN and originating somewhere on the Internet have similar characteristics. During these transmissions, the van transitioned the network in the following manner:

1. Nonpreferred path

2. Preferred path

3. Nonpreferred path

4. Preferred path

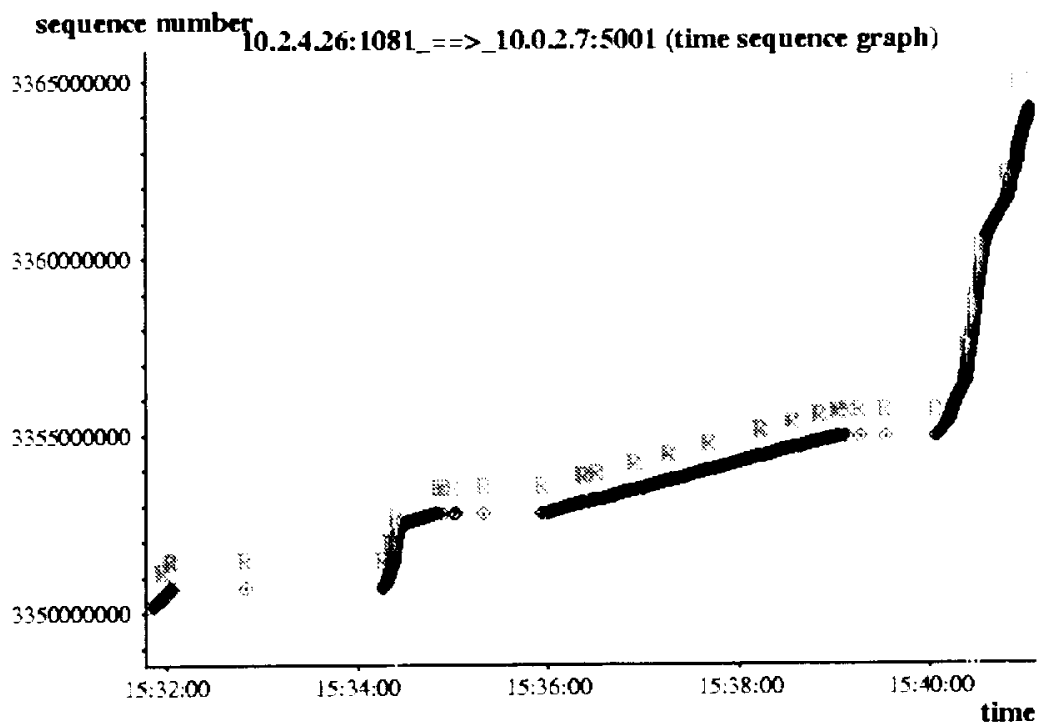

Figure 9. Source: Mobile Router LAN

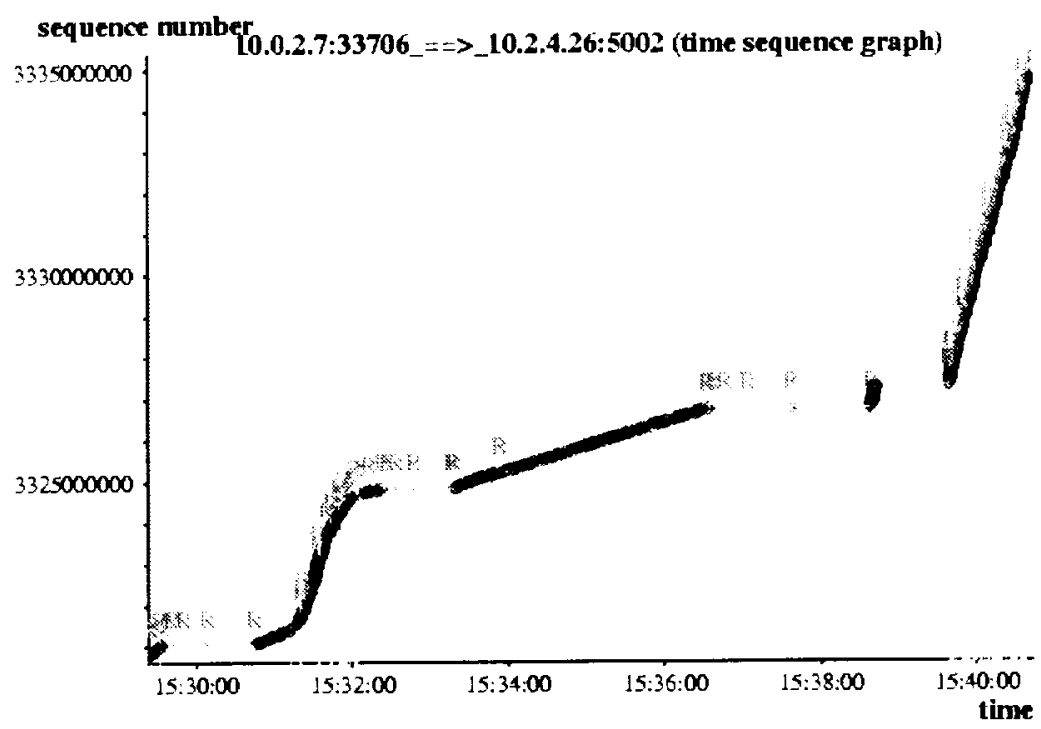

Figure 10. Destination: Mobile Router LAN 
Figure 11 is a blown-up section of figure 10 from time 15:30 to 15:32. Figure 11 shows the smooth handoff from the 128-kbps, 100-msec delay nonpreferred path to the 10-Mbps preferred path. Soon after utilizing the preferred path, we lost the preferred path link, as evidenced by the number of TCP retransmissions.

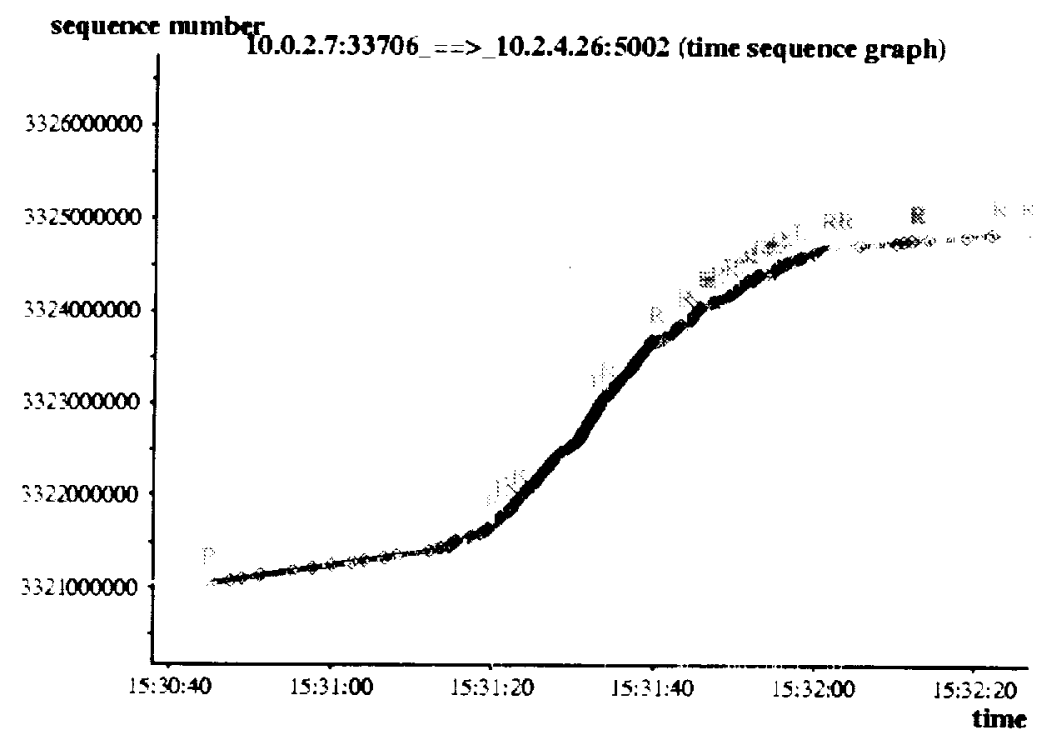

Figure 11. Smooth Handoff From Nonpreferred to Preferred Path

\section{SUMMARY}

We have described the mobile routing protocol and operation, the MR and some key configuration parameters. In addition, we provided a detailed description of the test network and the performance of transport protocols in dynamic routing environments. The results indicate that the current version of the MR code operates well, and handoffs are smooth between nonpreferred and preferred paths. In addition. the features available to manage handoffs can greatly improve performance, but they must be utilized judiciously with optimal settings varying between individual networks or network types.

\section{REFERENCE}

1. C. Perkins, RFC 2002 October 1996. 
Kent Leung is a senior software engineer specializing in IOS development at Cisco Systems, Inc., and an expert in mobile IP. His activities include contributing to IETF standards, developing features, filing numerous

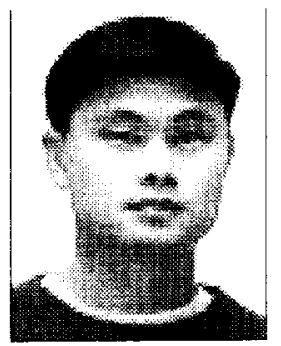
patent applications,

consulting, giving presentations, and training. Leung was the original and sole developer of the first commercial mobile-IP implementation at Cisco. He is now the technical lead on the development team as well as the developer for IOS Mobile Networks.

Dan Shell is a Senior Consulting Systems Engineer for Cisco Systems, Federal Operations. As the lead engineer in support of the CISCO/NASA Space Act Agreement for joint network research over high-delay and high-data-rate networks, Shell

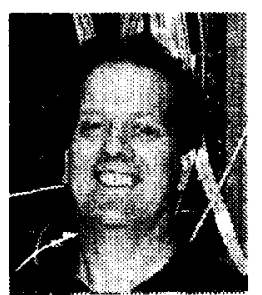
has been actively involved with NASA Glenn Research Center in researching IP over satellite and Internet nodes in space. In addition to supporting NASA Glenn, he is also responsible for the deployment of network solutions in the Federal sector, particularly with regard to wireless networks.

\section{Will Ivancic is a senior} research engineer at the NASA Glenn Research Center working in networking and advanced communication technology development. His work includes advanced digital and $R F$ design.

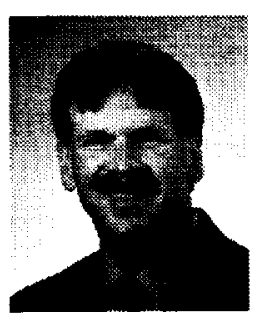
communications networks. satellite onboard processing, and system integration and testing. In addition, Ivancic has been responsible for the development of proof-of-concept onboard processing satellite equipment, ground terminal equipment, and numerous pieces of special test equipment. His recent research has been directed at applying commercial communication protocols to space and aeronautic base networks.

\section{David Stewart is a}

communication engineer at Verizon. He specializes in $R F$ and wireless communication networks. For the past 6 years, Stewart has supported advanced high-speed networking experiments on NASA's

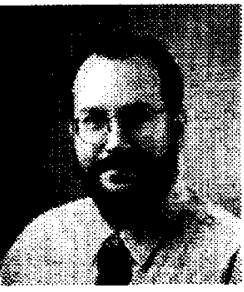
Advanced Communication Technologv Satellite, including support of the OCl2 high-data-rate terminals. His current work involves development and deployment of an MR testbed at Glenn, as well as deployment of early-field-trial aeronautic and maritime mobile networks.

\section{Terry Bell is a}

telecommunication network specialist for Lockheed Martin Global

Telecommunications. Over the past 7 years, Bell has supported Advanced Communication Technology

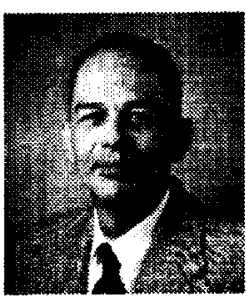
Satellite spacecraft operations, as well as a variety of satellite networking experiments. His recent responsibilities have included support of advanced protocol research for space and aeronautical-based networks.

Brian Kachmar is a senior software engineer of the Analex Corporation. Kachmar supported a miriad of advanced applications research experiments and demonstrations under the Advanced Communication

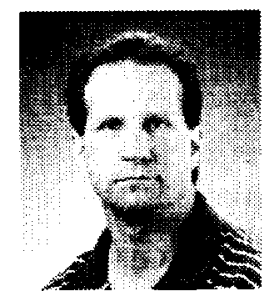
Technology Satellite experiments program, including unidirectional link routing evaluations, multicasting. and telemammography telemedicine. His current interests are in mobile IP and mobile networks, as well as support of research for NASA 's aeronautics safety and capacity programs. 
